\title{
Les temps verbaux dans la presse d'actualité historique
}

\author{
Mairi $^{1}$ McLaughlin ${ }^{*}$ \\ ${ }^{1}$ Department of French, University of California, Berkeley, 4125 Dwinelle Hall, UC Berkeley, \\ Berkeley CA 94720-2580, États-Unis
}

\begin{abstract}
Résumé. Cette enquête a pour objectif d'examiner l'usage des temps verbaux dans la presse française historique. Le travail se base sur un échantillon d'environ 1,000 instances de formes verbales finies extraites de la Gazette. L'échantillon couvre la période qui va de la parution de la Gazette en 1631 jusqu'à la Révolution en 1789. A travers une analyse quantitative de l'échantillon conjuguée à une analyse qualitative d'exemples particuliers, nous traçons l'évolution de la sélection des temps verbaux dans la presse d'actualité historique. Les résultats font état de changements touchant d'une part le genre journalistique et d'autre part la langue française pendant cette période.
\end{abstract}

\begin{abstract}
Verb tense in historical news discourse. This aim of this study is to analyse the verb tenses used in historical French news discourse. The work presented here is based on a sample of around 1000 tokens of finite verb forms taken from the Gazette. The sample covers the period from the founding of this newspaper in 1631 to the French Revolution of 1789. The study traces the evolution of verb tense selection in French news discourse through quantitative analysis of the sample and qualitative analysis of individual examples. The results highlight changes taking place during this period which affect the journalistic genre on the one hand and the French language on the other.
\end{abstract}

\section{Introduction}

Les chercheurs s'intéressent depuis longtemps à l'usage des temps verbaux dans la presse contemporaine. Citons, à titre d'exemple, une étude de l'usage du passé simple au $\mathrm{XX}^{\mathrm{e}}$ siècle par Herzog (1981) et les travaux de Labeau portant sur la sélection des temps verbaux dans des sous-genres journalistiques particuliers tels que le compte rendu sportif (2004, 2007) et l'article nécrologique (2009). Les nombreuses enquêtes à visée plutôt théorique concernant le soi-disant conditionnel journalistique se basent souvent sur des données tirées de la presse contemporaine ${ }^{i}$. Une question centrale pour comprendre le système temporel du discours journalistique est de savoir quel est le rapport exact entre le passé simple et le passé composé. Dans une perspective synchronique, l'intérêt de cette question est que les fonctions de ces deux tiroirs peuvent se chevaucher; leur sélection est

* Corresponding author : mclaughlin@berkeley.edu 
donc réglée par tout un réseau de facteurs dont les fonctionnements n'ont été que partiellement expliqués. Ceci a été amplement illustré par des études portant sur le français journalistique dont, par exemple, les travaux de Waugh et Monville-Burston (1986) et de Engel (1990). La perspective diachronique, pour sa part, met en évidence un remplacement - certes graduel et partiel - du passé simple par le passé composé. Citons, à titre d'exemple, une étude du genre épistolaire par Caron et Liu (1999).

La présente étude offre un examen de l'usage des temps verbaux dans la presse française historique. Le travail se base sur un corpus de périodiques historiques datant des $\mathrm{XVII}^{\mathrm{e}}$ et $\mathrm{XVIII}^{\mathrm{e}}$ siècles que nous avons construit pour le projet intitulé Origines et évolution du français journalistique: histoire d'un genre et l'histoire de la langue (McLaughlin en cours de préparation). L'échantillon est extrait de la presse d'actualité couvrant la période qui va de la parution en 1631 du premier périodique à avoir connu un succès à long terme, à savoir la Gazette, jusqu'à la Révolution en 1789. L'échantillon comprend presque 1,000 instances de formes verbales finies. A travers une analyse quantitative de l'échantillon conjuguée à une analyse qualitative d'exemples particuliers, on esquissera l'évolution de la sélection des temps verbaux dans la presse d'actualité historique. On s'intéressera surtout au rapport entre le passé simple et le passé composé. Il sera aussi possible de temps à l'autre de comparer nos résultats à des données concernant la presse française contemporaine. L'objectif de cette enquête est double. Premièrement, nous voulons approfondir notre compréhension de la presse française historique et de son rapport à la presse contemporaine. Deuxièmement, nous voulons contribuer aux études du passé simple et du passé composé dans la langue française en général et dans le discours journalistique plus particulièrement. La Partie 2 offre une description du corpus, les résultats sont présentés dans la Partie 3, et nous offrons une conclusion générale dans la Partie 4.

\section{Corpus}

L'objectif de cette partie est de présenter le corpus dont l'échantillon sous examen ici a été extrait. Le corpus a été construit entre 2012 et 2013 pour notre projet sur les origines et l'évolution du français journalistique (McLaughlin en cours de préparation). Le corpus représente les premiers deux siècles de la presse périodique de langue française. Les informations (manuscrites et imprimées) circulaient bel et bien avant le XVII ${ }^{\mathrm{e}}$ siècle mais ce n'est qu'à partir des premières décennies de ce siècle que la presse adopte un rythme de publication régulier et que les premiers périodiques de langue française sont fondés. La Gazette est la première publication périodique de langue française à avoir connu un succès à long terme. Sa première édition a paru le 30 mai 1631 et le Dictionnaire des journaux indique qu'elle dure de manière continue - quoiqu'avec certains changements de titre jusqu'en 1792 (Feyel 1991: 492). Notre corpus commence donc en 1632, la première année pour laquelle il y a une série complète d'éditions de la Gazette. La période représentée par le corpus se termine en 1789 avec la Révolution française à cause des changements majeurs qui ont touché la presse à cette époque ${ }^{\mathrm{ii}}$.

La Gazette fait partie du genre homonyme des gazettes qui publiaient les informations d'actualité aux siècles classique et postclassique. Elle consiste pour la plupart en une série de dépêches d'information écrites par des correspondants situés en général hors de Paris et les informations qu'elle publie sont officielles parce qu'elle opère sous le contrôle du roi. A côté de la Gazette, le corpus comprend quatre autres titres qui sont censés représenter différents sous-genres journalistiques qui, comme la Gazette, restent toutefois généralistes ${ }^{\mathrm{iii}}$. Le corpus comprend deux autres périodiques officiels importants du XVII ${ }^{\mathrm{e}}$ siècle, le Journal des savans, le premier périodique scientifique fondé en 1665, et le Mercure galant, une revue plus légère fondée en 1672. Il y a une deuxième gazette dans le corpus, la Gazette d'Amsterdam qui a été fondée en 1688 et qui représente l'importante 
presse de langue française extraterritoriale à cette époque. La dernière publication dans le corpus est le Journal de Paris, le premier quotidien de langue française qui a paru pour la première fois en 1777. Le corpus est présenté dans le Tableau 1. 
Tableau 1. Corpus général.

\begin{tabular}{|c|c|c|c|c|c|c|c|}
\hline Périodique & 1632 & $\mathbf{1 6 5 7}$ & $\mathbf{1 6 8 2}$ & $\mathbf{1 7 0 7}$ & $\mathbf{1 7 3 2}$ & $\mathbf{1 7 5 7}$ & $\mathbf{1 7 8 2}$ \\
\hline Gazette (1631) & jan & mar & mai & jui & sep & nov & jan \\
\hline Journal des savans (1665) & & & mai & jui & sep & $\underline{\text { nov }}$ & jan \\
\hline Mercure galant (1672) & & & mai & jui & sep & nov & jan \\
\hline Gazette d' Amsterdam (1688) & & & & jui & sep & $\underline{\text { nov }}$ & jan \\
\hline Journal de Paris (1777) & & & & & & & jan \\
\hline
\end{tabular}

L'immensité des données que représentent les cinq périodiques dans le corpus veut dire que nous avons dû employer deux concepts pour construire un corpus de taille manipulable. Le premier concept est celui de la génération qui a permis de choisir un an tous les 25 ans à partir de 1632, l'année pour laquelle il existe une série complète de la Gazette. Alors, nous analysons la langue de ces périodiques dans les années suivantes: 1632, 1657, 1682, 1707, 1732, 1757 et 1782. Ensuite nous avons adapté la notion de la semaine construite utilisée dans la recherche sur les médias pour choisir un mois à étudier pour chaque année représentée par le corpus. Pour chaque mois dans le corpus, nous analysons toutes les éditions de la Gazette, du Journal des savans et de la Gazette d'Amsterdam; pour maintenir une sorte d'équilibre entre les différents périodiques, nous n'examinons qu'un quart du matériel publié par le Mercure galant et par le Journal de Paris chaque mois. Au total, le corpus est de taille moyenne : il comprend 540,000 mots. Nous disposons de transcriptions complètes des mois soulignés dans le Tableau 1 qui représentent ensemble 395,000 mots.

La présente enquête se base sur un échantillon que nous avons extrait de ce corpus général pour l'examen des temps verbaux. Nous avons choisi de ne travailler que sur la Gazette et ceci pour deux raisons. Premièrement, la Gazette est la seule publication qui soit présente dès le début de la période et nous voulions privilégier la perspective diachronique. Deuxièmement, il était important de travailler sur la presse d'actualité pour faciliter la comparaison avec la presse contemporaine parce que la plupart des études linguistiques concerne les grands quotidiens d'information. L'échantillon consiste en 4 pages qui ont été choisies au hasard pour chaque année grâce à un générateur de nombres aléatoires. Nous avons extrait toutes les formes verbales finies et nous avons pu résoudre tous les cas d'ambiguïté éventuelle à travers le contexte ${ }^{\text {iv }}$. Au total, l'échantillon comprend 936 instances, un nombre suffisamment élevé pour pouvoir discerner un certain nombre de tendances générales en ce qui concerne la présence et l'évolution des différents temps verbaux.

\section{Résultats}

\subsection{L'évolution de l'usage des temps verbaux}


Cette première partie de l'étude offre une vue d'ensemble de la distribution des tiroirs verbaux dans la Gazette entre 1632 et 1782 . La distribution générale des tiroirs verbaux est présentée dans le Tableau 2 avec la fréquence relative entre parenthèses.

Tableau 2. Distribution des tiroirs dans la Gazette (1632-1782).

\begin{tabular}{|c|c|c|c|c|c|c|c|c|}
\hline & 1632 & 1657 & 1682 & 1707 & 1732 & 1757 & 1782 & TOTAL \\
\hline PRES $^{\mathrm{v}}$ & $\begin{array}{c}61 \\
(42.65) \\
\end{array}$ & $\begin{array}{c}31 \\
(47.69) \\
\end{array}$ & $\begin{array}{c}8 \\
(8.89) \\
\end{array}$ & $\begin{array}{c}16 \\
(14.81)\end{array}$ & $\begin{array}{c}22 \\
(23.40) \\
\end{array}$ & $\begin{array}{c}51 \\
(22.17) \\
\end{array}$ & $\begin{array}{c}54 \\
(26.21) \\
\end{array}$ & $\begin{array}{c}243 \\
(25.96) \\
\end{array}$ \\
\hline PC & $\begin{array}{c}28 \\
(19.58) \\
\end{array}$ & $\begin{array}{c}12 \\
(18.46) \\
\end{array}$ & $\begin{array}{c}16 \\
(17.78) \\
\end{array}$ & $\begin{array}{c}8 \\
(7.41) \\
\end{array}$ & $\begin{array}{c}18 \\
(19.15) \\
\end{array}$ & $\begin{array}{c}79 \\
(34.35) \\
\end{array}$ & $\begin{array}{c}66 \\
(32.04) \\
\end{array}$ & $\begin{array}{c}227 \\
(24.25) \\
\end{array}$ \\
\hline PS & $\begin{array}{c}18 \\
(12.59) \\
\end{array}$ & $\begin{array}{c}5 \\
(7.69) \\
\end{array}$ & $\begin{array}{c}33 \\
(36.67) \\
\end{array}$ & $\begin{array}{c}39 \\
(36.11) \\
\end{array}$ & $\begin{array}{c}24 \\
(25.53) \\
\end{array}$ & $\begin{array}{c}54 \\
(23.48) \\
\end{array}$ & $\begin{array}{c}21 \\
(10.19)\end{array}$ & $\begin{array}{c}194 \\
(20.73)\end{array}$ \\
\hline IMP & $\begin{array}{c}9 \\
(6.29) \\
\end{array}$ & $\begin{array}{c}5 \\
(7.69) \\
\end{array}$ & $\begin{array}{c}12 \\
(13.33) \\
\end{array}$ & $\begin{array}{c}21 \\
(19.44) \\
\end{array}$ & $\begin{array}{c}7 \\
(7.45) \\
\end{array}$ & $\begin{array}{c}16 \\
(6.96) \\
\end{array}$ & $\begin{array}{c}25 \\
(12.14) \\
\end{array}$ & $\begin{array}{c}95 \\
(10.15) \\
\end{array}$ \\
\hline PQP & $\begin{array}{c}1 \\
(0.7) \\
\end{array}$ & $\begin{array}{c}1 \\
(1.54)\end{array}$ & $\begin{array}{c}8 \\
(8.89) \\
\end{array}$ & $\begin{array}{c}10 \\
(9.26) \\
\end{array}$ & $\begin{array}{c}11 \\
(11.70) \\
\end{array}$ & $\begin{array}{c}18 \\
(7.83) \\
\end{array}$ & $\begin{array}{c}2 \\
(0.97) \\
\end{array}$ & $\begin{array}{c}51 \\
(5.45) \\
\end{array}$ \\
\hline PA & $\begin{array}{c}1 \\
(0.7) \\
\end{array}$ & & & & & & & $\begin{array}{c}1 \\
(0.11) \\
\end{array}$ \\
\hline COND & $\begin{array}{c}2 \\
(1.4) \\
\end{array}$ & & $\begin{array}{c}6 \\
(6.67) \\
\end{array}$ & $\begin{array}{c}4 \\
(3.70) \\
\end{array}$ & $\begin{array}{c}2 \\
(2.13) \\
\end{array}$ & $\begin{array}{c}1 \\
(0.43) \\
\end{array}$ & $\begin{array}{c}2 \\
(0.97) \\
\end{array}$ & $\begin{array}{c}17 \\
(1.82) \\
\end{array}$ \\
\hline $\mathbf{C P}$ & & & $\begin{array}{c}1 \\
(1.11) \\
\end{array}$ & $\begin{array}{c}1 \\
(0.93) \\
\end{array}$ & & $\begin{array}{c}1 \\
(0.43) \\
\end{array}$ & $\begin{array}{c}1 \\
(0.48) \\
\end{array}$ & $\begin{array}{c}4 \\
(0.43) \\
\end{array}$ \\
\hline FS & $\begin{array}{c}11 \\
(7.69)\end{array}$ & $\begin{array}{c}5 \\
(7.69) \\
\end{array}$ & $\begin{array}{c}2 \\
(2.22) \\
\end{array}$ & $\begin{array}{c}5 \\
(4.63) \\
\end{array}$ & $\begin{array}{c}9 \\
(9.57) \\
\end{array}$ & $\begin{array}{c}5 \\
(2.17) \\
\end{array}$ & $\begin{array}{c}24 \\
(11.65) \\
\end{array}$ & $\begin{array}{c}61 \\
(6.52) \\
\end{array}$ \\
\hline FP & $\begin{array}{c}4 \\
(2.8) \\
\end{array}$ & & & & & & & $\begin{array}{c}4 \\
(0.43) \\
\end{array}$ \\
\hline FA & & $\begin{array}{c}2 \\
(3.08) \\
\end{array}$ & & & & & & $\begin{array}{c}2 \\
(0.21) \\
\end{array}$ \\
\hline $\begin{array}{l}\text { Venir de } \\
+ \text { INF }\end{array}$ & & & & & $\begin{array}{c}1 \\
(1.06)\end{array}$ & $\begin{array}{c}2 \\
(0.87)\end{array}$ & $\begin{array}{c}2 \\
(0.97)\end{array}$ & $\begin{array}{c}5 \\
(0.53)\end{array}$ \\
\hline $\begin{array}{l}\text { SUBJ } \\
\text { PRES }\end{array}$ & $\begin{array}{c}4 \\
(2.8) \\
\end{array}$ & $\begin{array}{c}2 \\
(3.08) \\
\end{array}$ & $\begin{array}{c}2 \\
(2.22) \\
\end{array}$ & & & $\begin{array}{c}1 \\
(0.43)\end{array}$ & $\begin{array}{c}4 \\
(1.94) \\
\end{array}$ & $\begin{array}{c}13 \\
(1.39)\end{array}$ \\
\hline $\begin{array}{l}\text { SUBJ } \\
\text { IMP }\end{array}$ & & $\begin{array}{c}2 \\
(3.08) \\
\end{array}$ & $\begin{array}{c}1 \\
(1.11)\end{array}$ & $\begin{array}{c}3 \\
(2.78) \\
\end{array}$ & & $\begin{array}{c}2 \\
(0.87) \\
\end{array}$ & $\begin{array}{c}1 \\
(0.48) \\
\end{array}$ & $\begin{array}{c}9 \\
(0.96) \\
\end{array}$ \\
\hline $\begin{array}{l}\text { SUBJ } \\
\text { PASSÉ }\end{array}$ & $\begin{array}{c}3 \\
(2.1) \\
\end{array}$ & & & & & & $\begin{array}{c}3 \\
(1.46) \\
\end{array}$ & $\begin{array}{c}6 \\
(0.64) \\
\end{array}$ \\
\hline $\begin{array}{l}\text { SUBJ } \\
\text { PQP }\end{array}$ & $\begin{array}{c}1 \\
(0.7)\end{array}$ & & $\begin{array}{c}1 \\
(1.11)\end{array}$ & $\begin{array}{c}1 \\
(0.93)\end{array}$ & & & $\begin{array}{c}1 \\
(0.48)\end{array}$ & $\begin{array}{c}4 \\
(0.43)\end{array}$ \\
\hline TOTAL & $\begin{array}{c}143 \\
(100.00)\end{array}$ & $\begin{array}{c}65 \\
(100.00)\end{array}$ & $\begin{array}{c}90 \\
(100.00)\end{array}$ & $\begin{array}{c}108 \\
(100.00)\end{array}$ & $\begin{array}{c}94 \\
(100.00)\end{array}$ & $\begin{array}{c}230 \\
(100.00)\end{array}$ & $\begin{array}{c}206 \\
(100.00)\end{array}$ & $\begin{array}{c}936 \\
(100.00)\end{array}$ \\
\hline
\end{tabular}


Le Tableau 2 témoigne tout d'abord de la dominance de trois temps verbaux dans le corpus : le présent, le passé composé et le passé simple. Pris ensemble, ces trois tiroirs représentent plus de deux tiers $(71 \%)$ de toutes les formes verbales finies dans l'échantillon. Leur dominance s'explique par le fait que ces trois tiroirs sont les temps narratifs principaux dans la Gazette. Mais il faut noter que leur usage n'est pas stable à travers le temps. En fait, une comparaison de l'usage de ces trois temps verbaux permet d'identifier trois phases distinctes.

La première phase réunit les années 1632 et 1657 et elle se caractérise par la dominance du temps verbal du présent dont la fréquence relative est de $43 \%$ et de $48 \%$ respectivement. Comme l'illustrent les exemples (1) et (2), le présent remplit souvent la fonction du temps narratif à cette époque en rapportant les événements chronologiques successifs.

(1) De Rome, le 8. Decembre 1631.

BIEN que sa Saincteté ne se lasse point de travailler à la paix, les levées ne laissent pas de se continuer par toute l'Italie en grande diligence : notamment à Naples \& à Milan, où le Gouverneur de Sicile doit envoyer un regiment de gens de pied qu'il leue aussi à cette fin ${ }^{\text {vi }}$. (Gazette, Nouvelles ordinaires, 2 janvier 1632).

(2) De Rome, le 8. Decembre 1631.

TAndis que le Comte de Hatzféld met en estat les Troupes Impériales qui sont dans la Silésie, pour épier la marche des Transsilvains \& des Cosaques, le Comte Montécuculli, avec une partie de nos nouvelles levées, a ordre d'observer aussi la contenance des Turcs, qui s'avancent vers la Stirie \& la Carinthie, à dessein de s'ouvrir le passage de la Dalmatie (Gazette, 24 mars 1657).

Notons en passant que le présent est aussi un temps verbal fréquent dans la presse contemporaine. Citons, par exemple, une analyse de comptes-rendus sportifs par Labeau (2004 : 136) où $41.8 \%$ des formes verbales finies sont au présent. Neiger et TenenboimWeinblatt $(2016$ : 147) ont esquissé quatre types de présents associés à la production des nouvelles dans la presse contemporaine : «the present of the reporter while producing the item, the present of the editorial staff while processing it, the present of the material production of the item, and the more open present of its consumption ». Il nous semble que pour la plupart, le temps verbal du présent utilisé dans la Gazette renvoie au présent du correspondant.

La deuxième phase dans l'évolution de l'usage des temps verbaux correspond au milieu de la période, regroupant les années 1682, 1707 et 1732 où le passé simple est dominant. Cette phase est moins uniforme en ce que la dominance du tiroir préféré est moins nette, surtout pour l'année 1732. Néanmoins, comme on le montrera, il s'agit d'une rupture importante avec la phase précédente. Dans cette phase, les événements de la narration sont souvent rapportés au passé simple comme dans les exemples (3) et (4).

(3) Le Clergé s'assembla en la maniére accoûtumée le 4 , le 5 \& le 6 de ce mois, le matin \& le soir. La Séance du 4 au matin, fut entiérement occupée par le rapport que fit l'Archevesque d'Alby de la Commission dont il estoit le Chef, \& qui avoit esté chargée d'examiner les affaires de Tolose \& de Pamiers. (Gazette, 7 avril 1682).

(4) Le 6 de ce mois, le Roy fit faire l'exercice aux Regimens de Cavalerie du Camp de Villa Nova ; le 8 , ces Regimens combatirent en presence de sa Majesté ; \& après ce combat simulé, le Roy fut salué d'une decharge generale de l'artillerie. (Gazette, 6 septembre 1732).

Les exemples (3) et (4) se distinguent des exemples (1) et (2) par leur usage du passé simple pour rapporter les événements chronologiques successifs.

Il y a deux autres différences entre les exemples (1) et (2) et les exemples (3) et (4) que nous voulons souligner. Premièrement, il y a un changement de perspective. Dans la première phase, les correspondants avaient tendance à décrire ce qui se passait au moment 
où ils écrivaient et l'insertion du texte des dépêches dans la Gazette ne comprenait généralement pas de changements touchant les marqueurs déictiques. Dans la deuxième phase, les correspondants ont plutôt tendance à rendre compte d'événements passés et/ou le texte est sujet à des modifications plus extensives en ce qui concerne les marqueurs déictiques. L'année 1682 dans notre corpus semble donc refléter un moment important où il y a eu un changement des conventions touchant la présentation des informations. La deuxième différence concerne le type d'information fourni par les correspondants. Les exemples (3) et (4) se distinguent encore des exemples (1) et (2) par le fait de préciser la date à laquelle les événements ont eu lieu. Les dates précises sont quatre fois plus fréquentes dans l'année 1732 dans l'échantillon que dans l'année 1632. L'importance de ces deux différences est qu'elles permettent d'éclaircir la rupture avec la première phase. Dans les deux cas, on a affaire à des changements touchant le discours journalistique en général : un changement de perspective, et une augmentation de la précision. Et dans les deux cas, on a raison de croire que ces changements au niveau discursif auraient privilégié l'usage du passé simple. Premièrement, le fait de raconter les événements passés implique évidemment l'usage d'un tiroir du passé au lieu du présent. Même si le passé composé s'est déjà présenté comme concurrent du passé simple, le passé simple s'emploie toujours comme temps narratif dans les registres formels au XVII siècle, surtout pour rapporter des événements touchés par la soi-disant « règle des 24 heures » ${ }^{\text {vii }}$. Deuxièmement, la datation des événements aurait sans doute favorisé l'usage du passé simple, au moins au début de cette phase, car ce n'est qu'à cette époque que le passé composé a commencé à remplacer le passé simple après certains adverbes temporels (Caron et Liu 1999). Nous avons pu confirmer cette hypothèse en examinant toutes les instances dans l'échantillon entre 1682 et 1732 où une date précise est fournie par le correspondant : le passé simple est employé à l'exclusion absolue du passé composé dans ce contexte ${ }^{\text {viii }}$.

La dernière phase dans l'évolution de l'usage des temps verbaux comprend les années 1757 et 1782 où c'est le passé composé qui est dominant. Mais cette fois-ci, il ne semble pas que la préférence d'un nouveau tiroir soit la conséquence de l'évolution du genre journalistique. Il s'agit plutôt d'un changement linguistique. De nouveau, un examen des exemples particuliers montre que le temps verbal dominant a souvent pour objectif de rapporter les événements de la narration comme le montrent les exemples (5) et (6).

(5) On a fait le 2 de ce mois, dans l'Eglise des Augustins de cette Ville, un service solemnel pour les Officiers \& les Soldats morts au service de l'Impératrice Reine. Leurs Majestés Impériales y ont assisté avec toute la Cour. (Gazette, 26 novembre 1757).

(6) LE 2 de ce mois, le Comte de Montezan, Ministre plénipotentiaire de France, a célébré la naissance de Monseigneur le Dauphin par une fête publique, où il a fait éclater beaucoup de goût, de magnificence \& de variété. (Gazette, 22 janvier 1782).

Les exemples (5) et (6) sont révélateurs en ce qu'ils se distinguent nettement des exemples (3) et (4) puisque la datation précise des événements est accompagnée non pas par le passé simple mais par le passé composé. La dominance quantitative du passé composé dans cette dernière phase ainsi que la présence de tels exemples suggèrent que le passé composé a remplacé le passé simple dans ce contexte communicatif au milieu du XVIII ${ }^{\mathrm{e}}$ siècle. L'intérêt de ce constat réside dans le fait que l'enquête de Caron et Liu (1999) a mené à une datation différente de ce changement dans le genre épistolaire. En examinant le choix de tiroir verbal avec les adverbes hier, la veille et le lendemain, ils ont trouvé que le passé simple était largement majoritaire au XVII ${ }^{\mathrm{e}}$ siècle (ibid. : 43) mais qu'il y a eu une baisse « vertigineuse » (ibid. : 45) au siècle post-classique. Il est possible qu'on observe ici une différence dans la chronologie de ce changement linguistique dans les deux genres textuels différents. Il sera important d'examiner cette question plus en détail dans une étude future centrée sur les adverbes temporels examinés par Caron et Liu (1999). 
La dernière phase se caractérise donc par la dominance du passé composé et par une diminution importante de la fréquence du passé simple qui ne représente plus que $10.19 \%$ des formes verbales finies en 1782. On ne peut qu'être frappé par la relative rareté du passé simple dans la Gazette à la fin de la période sous examen. Même s'il n'est pas facile de faire des comparaisons quantitatives entre la presse historique et la presse contemporaine à cause des grandes différences génériques séparant la presse historique et contemporaine ${ }^{\mathrm{ix}}$, il est évident que la fréquence du passé simple n'était guère plus élevée à la fin du XVIII ${ }^{\mathrm{e}}$ siècle qu'il ne l'est aujourd'hui. En fait, dans certaines rubriques, qui, il faut bien l'avouer, sont peut-être celles qui favorisent son usage, le passé simple a pu être plus fréquent au $\mathrm{XX}^{\mathrm{e}}$ siècle qu'il ne l'était en général dans la Gazette en 1782. Citons, à titre d'exemple, une étude de compte-rendus sportifs par Labeau (2007 : 210) où la fréquence du passé simple dans Le Monde « se maintient aux alentours de 20\% [de l'année 1950] jusqu'à la fin des années soixante-dix ».

\subsection{L'alternance du passé simple et du passé composé}

Le fait qu'à partir du milieu du XVIII ${ }^{\mathrm{e}}$ siècle, les journalistes fassent un usage moderne du passé composé pour narrer les événements chronologiques soulève une question intéressante : est-ce que la pratique de l'alternance du passé simple et du passé composé considérée caractéristique du genre journalistique aux $\mathrm{XX}^{\mathrm{e}}$ et $\mathrm{XXI}^{\mathrm{e}}$ siècles - existait déjà au siècle post-classique ? On ne peut répondre que partiellement ici mais on voudrait signaler l'intérêt évident d'une étude plus approfondie à l'avenir. Beaucoup de chercheurs ont porté leur attention sur l'alternance du passé composé et du passé simple dans le français journalistique. Citons, à titre d'exemple, Monville-Burston et Waugh (1985), Waugh et Monville-Burston (1986), et Engel (1990, 1998). Ayres-Bennnett et Carruthers avec Temple (2001 : 176-78) présentent un résumé succinct du phénomène. Elles soulignent la multiplicité des facteurs de différents ordres qui régissent le choix de tiroir (Engel 1990) et, à la base des travaux de Waugh et Monville-Burston (1986), elles fournissent plusieurs exemples des effets discursifs/pragmatiques de l'alternance du passé composé et du passé simple : «a PS may be used to signal the formal limits of a text or part of a text » (AyresBennett et Carruthers avec Temple $2001: 177)$; " the PS can throw an event into relief» (ibid.) ; il peut y a voir « a 'zoom lens effect', where the mental camera seems to focus in on an event» (ibid.: 178); " the PC signals the first event in the narrative, which is then taken up in the PS » (ibid.); et «[t]he PS may even be used for backgrounding a piece of information, or inserting it in a parenthetical manner ».

Nous avons examiné toutes les dépêches qui datent des années 1757 et 1782 dans l'échantillon tiré de la Gazette pour tenter d'identifier des exemples éventuels des effets décrits par Ayres-Bennett et Carruthers avec Temple pour la langue de la presse contemporaine. Le Tableau 3 présente un résumé de la fréquence de paragraphes comprenant soit le passé composé, soit le passé simple, soit ces deux tiroirs dans les années 1757 et 1782 de l'échantillon. 
Tableau 3. Passé simple et passé composé dans la Gazette (1757 et 1782).

\begin{tabular}{|c|c|c|c|c|}
\hline & PS & PC & PS + PC & TOTAL \\
\hline $\mathbf{1 7 5 7}$ & $\begin{array}{c}7 \\
(14.89)\end{array}$ & $\begin{array}{c}31 \\
(65.96)\end{array}$ & $\begin{array}{c}9 \\
(19.15)\end{array}$ & $\begin{array}{c}47 \\
(100)\end{array}$ \\
\hline $\mathbf{1 7 8 2}$ & $\begin{array}{c}3 \\
(12)\end{array}$ & $\begin{array}{c}19 \\
(76)\end{array}$ & $\begin{array}{c}3 \\
(12)\end{array}$ & $\begin{array}{c}25 \\
(100)\end{array}$ \\
\hline Total & $\begin{array}{c}10 \\
(13.89)\end{array}$ & $\begin{array}{c}50 \\
(69.44)\end{array}$ & $\begin{array}{c}12 \\
(16.67)\end{array}$ & $\begin{array}{c}72 \\
(100)\end{array}$ \\
\hline
\end{tabular}

Le Tableau 3 montre de manière claire que dans la deuxième moitié du XVIII ${ }^{\mathrm{e}}$ siècle, la cooccurrence du passé simple et du passé composé dans le même paragraphe est l'exception plutôt que la règle. Dans la majeure partie des cas $(69.44 \%)$, c'est le passé composé et non pas le passé simple qui est utilisé. Ceci confirme de nouveau notre constat dans la Partie 3.1 concernant la dominance du passé composé dans la dernière moitié du XVIII ${ }^{\mathrm{e}}$ siècle. Le paragraphe reproduit sous (7) peut être considéré comme représentatif de l'écriture de la Gazette de cette période.

(7) On a fait le 2 de ce mois, dans 1'Eglise des Augustins de cette Ville, un service solemnel pour les Officiers \& les Soldats morts au service de l'Impératrice Reine. Leurs Majestés Impériales y ont assisté avec toute la Cour. (Gazette, 26 novembre 1757).

Quant à la cooccurrence du passé composé et du passé simple, au total il y en a 12 exemples. En comparant ces exemples aux fonctions énumérées par Ayres-Bennett et Carruthers avec Temple (2001 : 177-78), il semble que le seul cas de figure similaire soit celui où le PC « signals the first event in the narrative, which is then taken up in the PS ». Ceci est illustré par l'exemple (8).

(8) LE ROI \& le Prince Royal de Prusse sont arrivés ici le 25 pour y passer le temps du carnaval. Sa Majesté qui étoit accompagnée du Général de Moellendorff, fut, en arrivant, faire une visite à la Princesse Amélie, \& il y eut Cour extraordinaire au château, à l'occasion de l'arrivée du Roi. (Gazette, 18 janvier 1782).

L'échantillon comprend plusieurs autres exemples où le passé composé est utilisé en tête de paragraphe, suivi par le passé simple. On pourrait peut-être voir dans ces exemples un usage discursivo-pragmatique du passé composé pour marquer le début du paragraphe mais il nous semble que cet usage soit motivé plutôt par la fonction aspecto-temporelle du passé composé qui est d'indiquer un lien au présent. Ceci est illustré par l'exemple (9).

(9) Le Pere Louis Centurioni, Génois, Général des Jésuites, après une longue maladie, est mort à Castel-Gandolfe près de cette Ville le 2 du mois dernier, dans la soixantedouxiéme année de son âge, \& son corps fut transporté le lendemain ici. Après avoir été exposé pendant deux jours dans une chapelle de la Maison Professe des Jésuites, il

fut inhumé le 5 dans l'Eglise $d u$ Gesu, \& réuni à ses prédécesseurs dans la sépulture commune à tous les Généraux de l'Ordre. (Gazette, 5 novembre 1757).

L'usage du passé composé et du passé simple dans l'exemple (9) est similaire à l'usage de ces deux tiroirs dans des articles nécrologiques publiés au $\mathrm{XX}^{\mathrm{e}}$ siècle et décrits par Labeau (2009). Il se peut bien que l'alternance des tiroirs qui est associée au français journalistique contemporain ait eu ses débuts dans la presse historique aux $\mathrm{XVII}^{\mathrm{e}}$ et $\mathrm{XVIII}{ }^{\mathrm{e}}$ siècles mais elle ne peut aucunement être considérée un trait de ce genre à cette époque comme elle l'est de nos jours. 


\subsection{Les autres tiroirs}

L'objectif de cette partie de l'enquête est de faire des commentaires sur un certain nombre d'autres tiroirs qui sont présents dans la Gazette entre 1632 et 1782. Premièrement, il est à noter qu'à part les trois tiroirs dominants du présent, du passé composé et du passé simple, les journalistes se servent régulièrement dans leurs annonces et narratifs de trois autres temps verbaux : l'imparfait, le plus-que-parfait et le futur simple. L'usage du futur simple est particulièrement intéressant, surtout du point de vue générique. En examinant les exemples repérés, nous avons noté que le futur simple est utilisé pour annoncer les événements futurs. Ceci est illustré très clairement par l'exemple (10).

(10) Le Roy partira le 26 de ce mois pour aller à Cell où il fera la reveuë de deux Escadrons de Cavalerie; \& le 28 à Goerde, pour y prendre le divertissement de la chasse. Le Baron d'Hartoff, Chancelier de l'Electorat, retournera avec le Roy en Angleterre, \& le Baron de Mohr fera icy les fonctions de cette charge. (Gazette, 6 septembre 1732)

Nous voulons attirer l'attention sur l'usage illustré par (10) pour souligner le fait que rapporter les nouvelles n'implique pas toujours rapporter les événements passés; nous avons déjà vu dans la Partie 3.1 que les journalistes peuvent rendre compte d'événements en cours mais l'exemple (10) montre que même aux tous débuts de la presse périodique, les correspondants et journalistes annonçaient des événements qui allaient se passer à l'avenir. Ce constat vient confirmer l'intérêt d'une piste de recherche relativement récente sur le traitement du futur dans la presse. Neiger (2007) a développé un modèle des différents types d'actes spéculatifs qui peuvent se présenter, rangés selon le niveau de certitude/spéculation: "predictable future», "informed assessment», "speculative assessment» et «conjectured future » (ibid. : 314). En général, le type de spéculation impliqué dans notre échantillon se trouve vers le pôle plus certain. Ceci s'aligne bien sur ce que l'on sait déjà du contenu et du style de cette publication officielle. Comme le dit Feyel (1999: 13), "[j]usque dans les années 1770, la presse d'information française se vit interdire tout commentaire politique » donc on peut s'attendre à ce que les responsables de la Gazette aient évité la spéculation et se soient concentrés sur les informations officielles certaines.

Il est aussi intéressant de comparer l'usage du futur simple au futur périphrastique qui est beaucoup plus rare dans l'échantillon. Il y a 62 instances du future simple mais le futur proche ne figure qu'à 4 reprises. Il est notable que chacun de ces 4 exemples datent de 1632. Son usage au début du XVII ${ }^{\mathrm{e}}$ siècle n'est pas surprenant du point de vue de l'évolution de la langue puisque ses premières attestations datent du $\mathrm{XV}^{\mathrm{e}}$ (Rickard 1989: 75). C'est plutôt du point de vue générique que son usage nous intéresse. Comme l'illustre l'exemple (11), dans la plupart des cas, le futur proche se trouve dans le contexte d'une narration au présent.

(11) Les Princes \& autres Seigneurs qui sont icy, n'y engendrent point de melancholie. Ce ne sont que festins \& balets au tour du bouquet, en attendant la foire de S. Germain qui va rendre ceste liesse publique. (Gazette, 30 janvier 1632).

Le lien entre le futur proche et la narration au présent pourrait expliquer l'apparente rareté du futur proche dans les années subséquentes car, comme on l'a vu dans la Partie 3.1, la narration au présent devient plus rare après le milieu du XVII ${ }^{\mathrm{e}}$ siècle.

Examinons enfin une périphrase verbale qui suit un parcours différent dans l'échantillon, à savoir venir de + INF. Cette paraphrase ne s'emploie pas au début de la période mais commence à paraître plus régulièrement à partir de 1732 sans jamais atteindre une fréquence élevée ; venir de + INF ne parait que 5 fois dans l'échantillon. Etant donné que, comme le dit Vetters (2010: 292), le «processus de grammaticalisation de la construction » est "avancé » au XVII ${ }^{\mathrm{e}}$ siècle, sa parution dans l'échantillon n'est pas remarquable. Mais Vetters $(2010: 292)$ soulève la question de l'évolution du sens de cette 
périphrase. Les données qu'il présente indiquent que «le passage d'antérieur à passé perfectif a eu lieu entre le $X_{V I}{ }^{\mathrm{e}}$ et le $X X^{\mathrm{e}}$ siècle » et il est certain que la valeur du passé récent s'était déjà établie au $\mathrm{XIX}^{\mathrm{e}}$ siècle. Vetters cite comme preuves des exemples comme (12).

(12) Nous venons récemment de perdre encore une sœur charmante. (Lamartine, cité par Gougenheim 1929: 127, cité par Vetters 2010 : 293).

La rareté de cette périphrase dans notre échantillon empêche d'en faire des analyses plus poussées mais il est possible que sa fréquence quelque peu plus élevée à partir de 1732 reflète l'émergence de la valeur de passé récent. Cette valeur est certainement présente dans un exemple comme (13) où il s'agit d'un compte rendu d'une séance parlementaire.

(13) Le sieur Powis seconda l'Orateur qui venoit de traiter la matiere sur laquelle il devoit parler : il ajouta les observations les plus fortes à ce que venoit de dire Sir James (Gazette, 4 janvier 1782).

Les exemples qui datent de 1732 et de 1757 sont moins clairs mais il semble tout de même possible de leur associer une valeur de passé récent comme dans l'exemple (14).

(14) Le nommé Jacob, Ingenieur \& fils naturel du Duc de Riperda qu'on avoit arresté il y a quelque temps à Ceuta où il estoit allé pour lever le plan de cette place $\&$ pour seduire quelques Officiers de la garnison, a eu la question à Seville, \& l'on assure qu'il a declaré des choses très-importantes. On vient de faire partir pour cette place des provisions, des munitions de guerre \& plusieurs pieces de canon. (Gazette, 20 septembre 1732).

Ce qui privilégie la lecture du passé récent dans l'exemple (14) est l'urgence de la situation. Une étude plus approfondie de l'usage de cette périphrase verbale dans les périodiques historiques pourrait s'avérer utile pour éclaircir encore plus cette question du passage du sens antérieur au sens du passé récent.

\section{Conclusion}

L'objectif de cette enquête était d'examiner l'usage des tiroirs verbaux dans la presse historique de langue française. Nous avons exploré la distribution générale des différents tiroirs dans une perspective diachronique. A travers une analyse quantitative et qualitative de la Gazette, une publication qui représente la presse d'actualité des XVII ${ }^{\mathrm{e}}$ et XVIII ${ }^{\mathrm{e}}$ siècles, nous avons pu tracer l'histoire de l'usage des différents temps verbaux. Les résultats de l'enquête ont jeté de la lumière sur plusieurs aspects de l'écriture journalistique historique. Nous avons identifié un changement générique important en 1682 qui consiste premièrement en une augmentation de la précision des informations grâce à une inclusion plus régulière des dates précises des événements rapportés et adeuxièmement en une nouvelle perspective liée au rapport des événements passés au lieu des événements en cours. L'enquête a aussi jeté de la lumière sur le rapport entre la presse historique et la presse contemporaine ; il est surtout intéressant de noter que l'annonce d'événements futurs est déjà une pratique courante à cette époque. Enfin les résultats ont aussi contribué aux recherches concernant l'évolution du passé simple et du passé composé en soulevant la possibilité que ce changement ait eu lieu dans le contexte d'adverbes temporels un peu plus tard dans la presse d'actualité que dans le genre épistolaire. Néanmoins, comme c'est souvent le cas, le travail présenté ici souligne la nécessité de recherches supplémentaires dans ce domaine, surtout celles portant sur d'autres périodiques de langue française des siècles classique et postclassique.

\section{Références bibliographiques}

Ayres-Bennett, W., J. Carruthers et R. Temple. (2001). Studies in the modern French language : problems and perspectives. Harlow : Longman. 
Caron, P. et Y.-C. Liu. (1999). Nouvelles données sur la concurrence du passé simple et du passé composé dans la littérature épistolaire. L'information grammaticale, 82, 38-50.

Duranton, H., C. Labrosse et P. Rétat (1992). Les gazettes européennes de langue française : XVII et XVIII ${ }^{e}$ siècles. Saint-Étienne : Publications de l'Université de Saint-Étienne.

Engel, D. (1990). Tense and text : a study of French past tenses. London/New York : Routledge.

Engel, D. (1998). A perfect piece ? The present perfect and passé composé in journalistic texts. Belgian journal of linguistics, 12, 129-147.

Feyel, G. (1991). Gazette de France. Dans Dictionnaire des journaux 1600-1789, dir. par J. Sgard, 2 vols. Paris : Universitas, Vol. I : 443-49.

Feyel, G. (1999). La presse en France des origines à 1944. Paris : Ellipses.

Feyel, G. (2000). L'annonce et la nouvelle: la presse d'information en France sous l'ancien régime (1630-1788). Oxford : Voltaire Foundation.

Fournier, N. (2002). Grammaire du français classique. Paris : Belin.

Gougenheim, G. (1929). Etude sur les périphrases verbales de la langue française. Paris : Nizet.

Haffemayer, S. (2002). L'information dans la France du XVII siècle: la Gazette de Renaudot, de 1647 à 1663, Paris: H. Champion.

Herzog, C. (1981). Le passé simple dans les journaux du XX'e siècle. Berne : Editions Francke.

Kronning, H. (2012). Le conditionnel épistémique : propriétés et fonctions discursives. Langue française, 173(1), 83-97.

Labeau, E. (2004). Le(s) temps du compte rendu sportif. Journal of French language studies, 14(2), $129-48$.

Labeau, E. (2007). Et un, ou deux, ou trois? Les temps-champions du reportage sportif depuis 1950. Cahiers chronos, 16, 203-33.

Labeau, E. (2009). Le PS: cher disparu de la rubrique nécrologique ?. Journal of French language studies, 19, 61-86.

McLaughlin, M. (en cours de préparation) Les origines et l'évolution du français journalistique : histoire d'un genre et l'histoire de la langue.

Monville-Burston, M. et L. Waugh. (1985). Le passé simple dans le discours journalistique. Lingua, $67(2 / 3), 121-170$.

Neiger, M. (2007). Media oracles: the cultural significance and political import of news referring to future events. Journalism, 8(3), 309-321.

Neiger, M. et K. Tenenboim-Weinblatt. (2016). Understanding journalism through a nuanced deconstruction of temporal layers in news narratives. Journal of communication, 66, 139-160.

Rickard, P. (1989). A history of the French language. $2^{\text {ème }}$ édn. London : Hutchinson \& Co.

Sgard, J. (dir.). (1991). Dictionnaire des journaux 1600-1789, 2 vols. Paris : Universitas.

Vetters, C. (2010). Développement et évolution des temps du passé en français: passé simple, passé composé et venir de + infinitif. Dans Temps, aspect et modalité en français, dir. par E. Moline et C. Vetters. Amsterdam : Rodopi, 277-298.

Vincent, M. (2005). Le Mercure galant : présentation de la première revue féminine d'information et de culture, 1672-1710. Paris : H. Champion.

Vittu, J.-P. (1988). Diffusion et réception du Journal des savants de 1665 à 1714. Dans La Diffusion et la lecture des journaux de langue française sous l'Ancien Régime, Actes du colloque international, Nimègue, 3-5 juin 1987, dir. par H. Bots. Amsterdam/Maarssen : APA - Holland University Press, 167-77. 
Waugh, L. R. et M. Monville-Burston. (1986). Aspect and discourse function: the French simple past in newspaper usage. Language : journal of the Linguistic Society of America, 62 (4), 846-877.

\footnotetext{
${ }^{\mathrm{i}}$ Voir par exemple Kronning (2012).

ii Pour une histoire générale de la presse française, voir Feyel (1999).

iii Pour des informations supplémentaires sur tous les périodiques dans le corpus, voir Vittu (1988), Sgard (1991), Duranton, Labrosse et Rétat (1992), Feyel (1999, 2000), Haffemeyer (2002) et Vincent (2005).

${ }^{\text {iv }}$ Citons à titre d'exemple la forme dit qui représente la troisième personne du singulier du verbe dire pour soit le présent soit le passé simple.

${ }^{\vee}$ La plupart des abréviations utilisées pour les tiroirs verbaux sont tirées de Labeau (2004: 136). $\mathrm{PRES}=$ présent, $\mathrm{PC}=$ passé composé, $\mathrm{PS}=$ passé simple, $\mathrm{IMP}=$ imparfait, $\mathrm{PQP}=$ plus-que-parfait, $\mathrm{PA}=$ passé antérieur, $\mathrm{COND}=$ conditionnel, $\mathrm{CP}=$ conditionnel du passé, $\mathrm{FS}=$ futur simple, $\mathrm{FP}=$ futur proche, $\mathrm{FA}=$ futur antérieur, $\mathrm{INF}=$ infinitif, $\mathrm{SUBJ}=$ subjonctif.

${ }^{\mathrm{vi}}$ Les exemples ont été transcrits avec des modifications minimales : les $s$ longs ont été remplacés par $s$ et l'usage de $u / v$ et de $i / j$ suit l'usage moderne.

${ }^{\text {vii }}$ Comme l'explique Fournier (2002:398) : «L'emploi du passé simple et du passé composé se trouve réglé par un principe purement référentiel, celui de la 'nuit entre deux', selon lequel les événements au delà de la nuit sont exprimés au passé simple et ceux en deçà au passé composé ». viii Sur un total de 31 dates précises indiquées dans l'échantillon pour les années 1682, 1707 et 1732, il y a 2 instances du futur simple, 1 instance du plus-que-parfait et 28 instances du passé simple.

${ }^{\mathrm{ix}}$ Voir McLaughlin (en cours de préparation, ch. 2).
} 